\title{
An innovative model of a non-relativist universe
}

\author{
Olga Baryshnikova ${ }^{1}$, Catherine Sapelnik ${ }^{1}$, and Lyudmila Fedoseeva ${ }^{1,{ }^{*}}$ \\ ${ }^{1}$ Don State Technical University, Gagarin Square 1, Rostov-on-Don 344003, Russia
}

\begin{abstract}
The paper investigates a model of the local Universe based on the concepts of nonrelativistic physics. The space-time boundaries of its applicability are estimated. The finiteness of the Universe in space and time arising from the analysis of the field of forces and energy fields of the Universe is affirmed. The necessity of the existence of "dark matter" in the proposed model is shown. On the basis of the presented model (using the modern values of the Hubble parameter and the density of the substance of the Universe), the following are estimated: the age of the Universe; the mass of its substance; period of her life cycle; acceleration of the movement of matter, etc. The dependences of the density of matter (and its minimum value), the radius of the Universe (and its maximum value), and the Hubble parameter on time are obtained. The position of the Milky Way galaxy in the Universe has also been estimated. It is shown that the law of conservation of energy in its classical formulation is not applicable to the Universe as a whole. It is argued that after a while the laws of classical physics will be applicable to the entire Universe as a whole.
\end{abstract}

\section{Introduction}

Since the earliest civilizations, there is an infinite variety of models of the universe. And this is not surprising, since people have always been interested in the world in which they live. Among the scientifically based models, we note the model of an infinite, stationary Universe proposed by I. Newton (1687) [1], A. Einstein's model of stationary but finite Universe (1917) based on general relativity [2]. According to the same theory, A. Friedman proposed a model of the expanding Universe [3]. Later, E. Hubble experimentally proved the expansion of the Universe [4]. Then G. Gamow proposed the "Hot Universe" hypothesis, which later turned into the Big Bang theory [5]. The discovery of the relict radiation has confirmed both the model of the expanding Universe and the Big Bang theory $[6,7,8]$. Further, the concept of "dark matter", first predicted in [9]. In addition, it was shown that the Universe is expanding with acceleration [10, etc.]. The modern model of the universe is $\Lambda \mathrm{CDM}$.

However, modern models of the Universe cannot give an unambiguous answer to the question about its future. In addition, modern models are very complex mathematically due to the need to use general relativity. Therefore, there is a need to create a model that would describe the global characteristics of the Universe and their dependence on time, without going into a detailed description of its structure.

\footnotetext{
${ }^{*}$ Corresponding author: fedoseevvb@gmail.com
} 
According to modern concepts, the Universe expands from a certain initial state, in which the laws of classical physics are obviously inapplicable. But as the Universe expands, regions appear in it that are fairly accurately described by the laws of nonrelativistic physics. For example, within our galaxy, a part of the Universe is described quite accurately by the laws of nonrelativistic physics, excluding small corrections in the behavior of the orbit of Mercury. One can imagine that as the Universe expands, these regions will increase until they cover the entire Universe. This part of the Universe, in which the laws of nonrelativistic physics are valid, we will name, following Ya.B. Zeldovich, the local Universe, and compose a mathematical model for this part of the Universe. Next, we will estimate the range of applicability of the model.

In such a setting, it seems perfectly reasonable to develop a model of the Universe based on classical concepts.

\section{Formulation of the problem}

Let's imagine the substance of the local Universe in the form of an ideal gas. Since this is a gas of the Universe, it must possess the properties of the Universe: isotropy and homogeneity, which implies the independence of the gas density from spatial coordinates. To describe the motion of such an ideal gas, we use the Euler equations:

$$
\left\{\begin{array}{c}
\frac{\partial v}{\partial t}+(\boldsymbol{v} \nabla) \boldsymbol{v}=-\frac{1}{\rho} \nabla P+\boldsymbol{g} \\
\frac{\partial \rho}{\partial t}+\operatorname{div}(\rho \boldsymbol{v})=0
\end{array}\right.
$$

To these equations it is necessary to add the thermodynamic equation of state of the substance of the Universe $-P=n k T \sim \rho T$. The density of a substance does not depend on $\mathrm{r}$, so $\nabla P \sim \nabla T$. But since there is no data yet on the temperature gradient in the Universe, we will not further consider the thermodynamic component.

Also, to these equations it is necessary to add the Hubble equation, which describes the property of our Universe. Thus, system (1) will take the form:

$$
\left\{\begin{array}{c}
\frac{\partial \mathbf{v}}{\partial \mathrm{t}}+(\mathbf{v} \nabla) \mathbf{v}=\mathbf{g} \\
\frac{\partial \rho}{\partial \mathrm{t}}+\operatorname{div}(\rho \mathbf{v})=0 \\
\mathbf{v}=\mathrm{Hr}
\end{array}\right.
$$

All vectors of system (2) are directed along the radius; therefore, in what follows, we omit the vector sign. Note that the left-hand side of the first equation of system (2) is the total derivative of the velocity with respect to time.

Because $\rho \neq f(r)$, but $\rho=f(t)$, so $\operatorname{div}(\rho v)=\rho \operatorname{div}(v)$. The velocity vector has only one radial component, therefore,

$$
\frac{\partial \rho}{\partial t}+\operatorname{div}(\rho v)=\frac{d \rho}{d t}+\rho 3 H
$$

The term $\boldsymbol{g}$ is the force of gravity acting on a unit of mass from the side of the gravitational field of the Universe itself, therefore $g=-G \frac{m}{r^{2}}=-\frac{4 \pi}{3} G \rho r$, where $m-$ the mass of the universe enclosed within a sphere of radius $r$. As a result of all transformations, system (2) takes the form: 


$$
\left\{\begin{array}{c}
\frac{d v}{\partial t}=g \\
\frac{d \rho}{d t}+3 \rho H=0 \\
v=H r
\end{array}\right.
$$

Eliminating the velocity from the equations of system (3), we find

$$
\left\{\begin{array}{c}
H^{2}+\frac{d H}{d t}=-\frac{4 \pi}{3} G \rho \\
\frac{d \rho}{d t}+3 \rho H=0
\end{array}\right.
$$

Similar equations were written earlier [11], but no attempts were made to integrate it.

Eliminating the parameter $\mathrm{H}$ from this system, we obtain one equation for determining the density of the substance of the Universe

$$
\frac{d^{2} \rho}{d t^{2}}=\frac{2}{\rho}\left(\frac{d \rho}{d t}\right)^{2}+4 \pi G \rho^{2}
$$

For the possibility of obtaining numerical estimates of the solution, we use the minimum number of known parameters of our Universe at the present time: Hubble parameter $-H_{0}[12]$ and the density of the Universe substance $-\rho_{0}$ [13].

$$
H_{0}=10^{-18}\left(\mathrm{c}^{-1}\right) ; \quad \rho_{0}=10^{-26}\left(\mathrm{~kg} / \mathrm{m}^{3}\right) .
$$

In equation (4), we pass to the dimensionless variables: $q=\rho / \rho_{0}$ and $\tau=t \cdot H_{0}$ :

$$
\frac{d^{2} q}{d \tau^{2}}=\frac{2}{q}\left(\frac{d q}{d \tau}\right)^{2}+A q^{2}
$$

Here the dimensionless parameter $A$ is determined by the expression:

$$
A=\frac{4 \pi G}{H_{0}^{2}} \rho_{0} \approx 0,08
$$

\section{The solution of the problem}

The solution to differential equation (6) has the form:

$$
q=\frac{2}{-A \tau^{2}+2 C \tau-2 C_{1}}
$$

Based on the fact that the time is considered to be positive, the constant of integration $C_{1}$ must be set equal to zero: $C_{1}=0$. In this case:

$$
q=\frac{2}{-A \tau^{2}+2 C \tau}=\frac{2}{\tau(2 C-A \tau)}
$$

From (8) it follows that the density of the substance of the universe changes from $\infty$ at $\tau=0$, to $\infty$ at $\tau=\tau_{2}=2 C / A$, passing through the minimum at $\tau=\tau_{m}=C / A$. Moreover, at the minimum point $q_{\text {min }}=\frac{2 A}{C^{2}} \neq 0$. Consequently, the Universe will complete a cycle of expansion-contraction and at the same time will not dissipate in the surrounding space, i.e. gravitational forces will compress the currently expanding Universe.

The Hubble parameter is determined from the second equation of the system (3) 


$$
H=-\frac{1}{3 \rho} \frac{d \rho}{d t}=-\frac{H_{0} \rho_{0}}{3 q \rho_{0}} \frac{d q}{d \tau}=-\frac{H_{0}}{3 q} \frac{d q}{d \tau}
$$

Finding the derivative $\frac{d q}{d \tau}$, we obtain the dependence of the Hubble parameter on time:

$$
H=\frac{2 \cdot H_{0}}{3} \frac{C-A \tau}{-A \tau^{2}+2 C \tau}
$$

Let us denote by $\tau_{0}$ время, the time corresponding to the present moment, i.e. the moment at which $\rho=\rho_{0}$, (and correspondingly $q=1$ ) and $H=H_{0}$. In this case, using (8) and (9), we obtain a system of two equations to determine two unknowns $-\tau_{0}$ and $C$ :

$$
\left\{\begin{array}{c}
1=\frac{2}{-A \tau_{0}^{2}+2 C \tau_{0}} \\
1=\frac{2}{3} \frac{C-A \tau_{0}}{-A \tau_{0}{ }^{2}+2 C \tau_{0}}
\end{array}\right.
$$

Positive solution to this system has the form:

$$
\tau_{0}=\frac{1}{2} \frac{-6+2 \sqrt{9+2 A}}{A}, \quad C=\sqrt{9+2 A}
$$

The maximum limits of variation of the parameter A for our Universe from 0 to 1 . Within this interval $\tau_{0}$, changes very smoothly. So, $\lim _{A \rightarrow 0} \tau_{0}=1 / 3=0.3333$, at the same time $\tau_{0}(1)=0.3166$. Therefore, for further calculations, we take $\tau_{0}=1 / 3$ and $C=3$.

\section{Solution analysis}

Thus, the maximum age of the universe can be $T_{0}=\tau_{0} / H_{0}=1 / 3 H_{0}=10.58$ billion years. The total time of our universe $T_{c}$ (cycle period) $\mathrm{v}(8)$, i.e $\tau_{2}=2 C / A$. Using the found value $C$, find

$$
T_{c}=\frac{\tau_{2}}{H_{0}}=\frac{2 C H_{0}}{4 \pi G \rho_{0}}
$$

Substituting numerical values, we find: $T_{U}=2338$ billion years. Thus, to date, our universe has existed for only 0,0045 part of all the time it should have been exist.

The minimum density of a substance that it reaches through $\tau=\tau_{m}=\frac{C}{A}=$ 1169 billion years, will be equal:

$$
q_{\min }=\frac{2 A}{C^{2}}=2 \frac{4 \pi G \rho_{0}}{9 H_{0}^{2}} \approx 0.018
$$

Therefore $\rho_{\text {min }}=0,018 \rho_{0}=1.8 \cdot 10^{-28}\left(\mathrm{~kg} / \mathrm{m}^{3}\right)$. The $q$ from $\tau$ dependency graph constructed according to formula (8) is shown at the figure 1 .

It follows from the graph at figure 1 that the Universe spends most of its time in a deeply rarefied state. The solution outside the roots corresponds to the negative density of the substance, which has no physical meaning, so we will not consider it. 


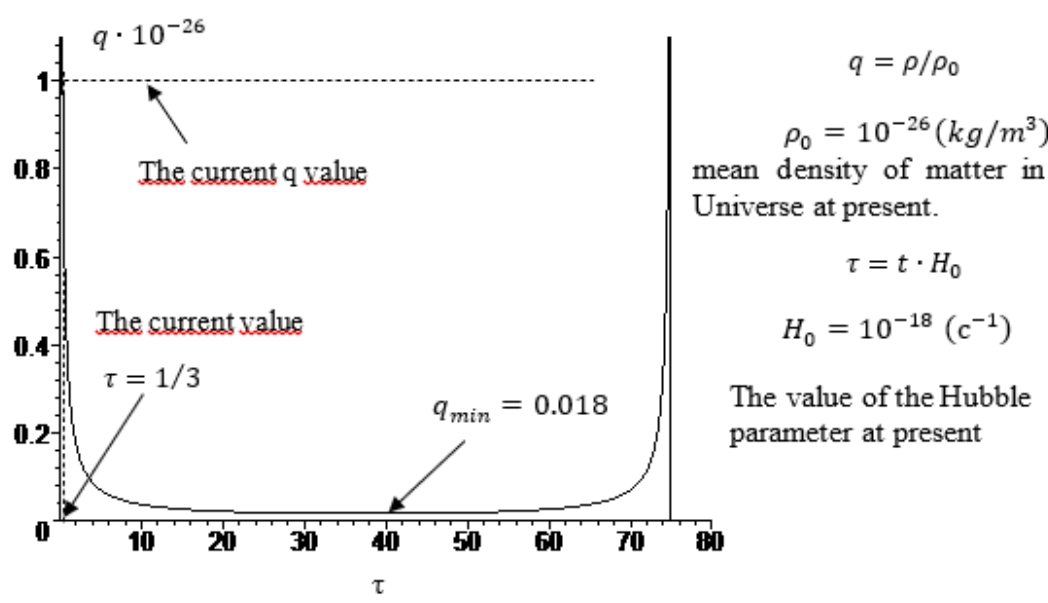

Fig. 1. Graph of dependence of the dimensionless density of matter in the Universe $q$ from dimensionless time $\tau$.

The dependence of the Hubble parameter on time is graphically shown at figure 2.

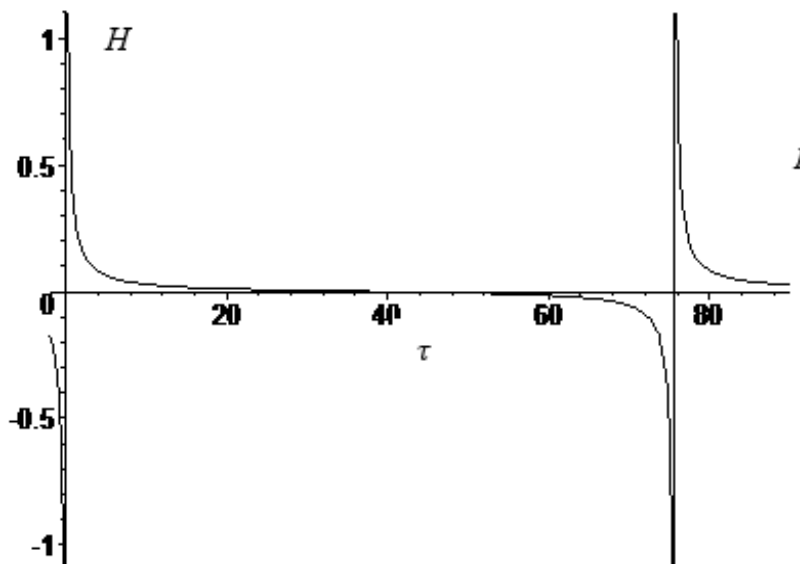

\section{$H$ - Hubble parameter}

$H_{0}$ - the Hubble parameter at present

$$
\tau=t \cdot H_{0}
$$

Fig. 2. Graph of dependence of the Hubble parameter on dimensionless time $\tau$.

We see that the Hubble parameter is rapidly decreasing during the period.

The Universe while staying positive. In the middle of the life cycle of the Universe, it becomes zero, and then increases again, being already negative. Similarly, the speed of movement of the substance of the Universe will change in time. At the beginning, it is positive and close to the speed of light (which corresponds to the expansion of the Universe, then it becomes equal to zero (the expansion process is over) and then it increases again, but already with a negative sign (the speed is directed to the center of the Universe).

Express the radius of the Universe $\mathrm{R}$ through its mass $\mathrm{M}$ :

$$
R=\sqrt[3]{\frac{3 M}{4 \pi \rho}}
$$

From (12) it follows that the Universe is expanding ( $\rho$ decreases), but not indefinitely, since the density of matter $\rho$ decreases not to zero, but to $\rho_{\text {min }}$, defined by the ratio (11). 
Consequently, its substance cannot go beyond itself. Therefore, from this point of view, the Universe is a "black hole".

But for the Universe to really be a "black hole", it is necessary that the energy ratio be fulfilled. During the period of maximum expansion, the kinetic energy of the Universe is zero, therefore, its potential energy must be greater than (or equal to) its rest energy.

$$
\frac{3}{5} G \frac{M^{2}}{R_{\max }} \geq M c^{2}
$$

Substituting here the value for $\rho_{\min }$ from (11), of the parameter A from (7), we obtain the following relation:

$$
M \rho_{0} \geq 0.38 \cdot 10^{28}
$$

Hence it follows that in order for the model of the Universe to really be a "black hole", it is necessary that its mass satisfies the following inequality:

$$
M \geq 3.8 \cdot 10^{53}(\mathrm{~kg})
$$

According to some data [14], the mass of the Universe exceeds $1053 \mathrm{~kg}$. In further calculations we will consider $M=10^{54}(\mathrm{~kg})$.

Substituting the value of $\mathrm{M}$ in (12), we find the dependence of the radius of the Universe on time:

$$
R=\sqrt[3]{\frac{3 \cdot 10^{54}}{4 \pi \cdot 10^{-26} \cdot q}}=2,87 \cdot 10^{26} \cdot q^{-1 / 3}
$$

Here $q$ determined by the formula (8). Note that at the moment $q\left(\tau_{0}\right)=1$, therefore the radius of the Universe at the moment is $R \approx 2.87 \cdot 10^{26}(\mathrm{~m})$. This figure is most often found in the literature.

Dependency graph of $R \cdot 10^{-26}$ on dimensionless time, constructed by formula (13), is shown in Figure 3.

We see that the radius of the Universe changes from zero to zero (at the beginning of the expansion and at the end of the expansion) passing through a maximum equal to $\approx 11 \cdot 10^{26}(\mathrm{~m})$. The graph is non-linear, so the rate of increase in distances depends on time.

Further, according to the first equation of system (3), the acceleration of the motion of the substance of the Universe $a$ is:

$$
a=\frac{d v}{d t}=-\frac{4 \pi}{3} G \rho r
$$

Hence it follows that acceleration is always negative, i.e. directed towards the center of the universe. This is not surprising since the acceleration is caused only by the force of gravity. In addition, acceleration, like speed, is also directly proportional to the spatial coordinate $r$. Therefore, the dependence of acceleration on time is similar to the dependence of density $q$, i.e similar to the graph at Figure 1 (up to a sign).

Consequently, in the middle of the life of the Universe, the acceleration is minimal and its value for the peripheral regions can be estimated as:

$$
w_{\min }=\frac{4 \pi}{3} G \rho_{\min } R
$$




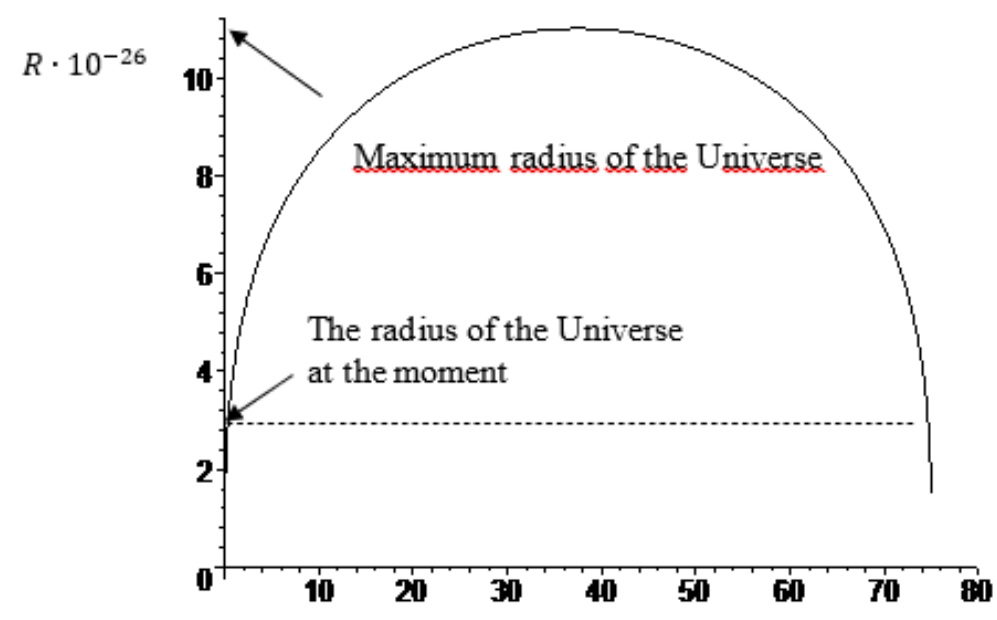

Fig. 3. Dependence of the radius of the Universe on dimensionless time $\tau$.

Substituting values for $\rho_{\min }$ and $R$, find that $w_{\min }=5.5 \cdot 10^{-11}\left(\mathrm{~m} / \mathrm{s}^{2}\right)$

Consider the speed of motion of the substance of the Universe. According to the third equation of system (2), we write

$$
v=H r
$$

Find the surface of space-time, where this speed is equal to the speed of light:

$$
r=\mathrm{c} / H
$$

Substitute the expression for $H$ from (9)

$$
r=\mathrm{c} / H=\frac{3 \mathrm{c}}{2 H_{0}} \frac{-A \tau^{2}+2 C \tau}{C-A \tau}
$$

Having made numerical calculations, we get:

$$
r=4.5 \cdot 10^{26} \cdot \frac{-A \tau^{2}+2 C \tau}{C-A \tau}
$$

At Figure 4, in some axes, we will depict graphs of the dependence of the radius of the Universe on time $-R$ formula (13), line where the speed of matter is equal to the speed of light $v=c$ formula (17), and the line on which the speed of matter is equal to one tenth of the speed of light $v=0.1 \cdot c$, i.e the same formula, but divided by ten. In this case, the region near $\tau=0$.

It follows from the figure that in the initial period of the life of the Universe, practically all matter moved at speeds close to the speed of light. But then, gradually, internal regions began to appear, in which matter moved at a speed lower than the speed of light. This area increases over time and will gradually encompass the entire universe. Solving together equations (13) and (16) (at $v=0.1 \cdot c$ ), we find what will have been happen at $\tau=$ 8 or $t=254$ billion years. That is, after this time, the laws of classical physics will be applicable to the entire Universe with a high degree of accuracy.

If we assume that there are no material objects in our galaxy moving at a speed $v>$ $0.1 \cdot c$, then the Milky Way has an absolute coordinate $r=0.3 \cdot 10^{26}(\mathrm{~m})$, or about onetenth of the current radius of the Universe. 


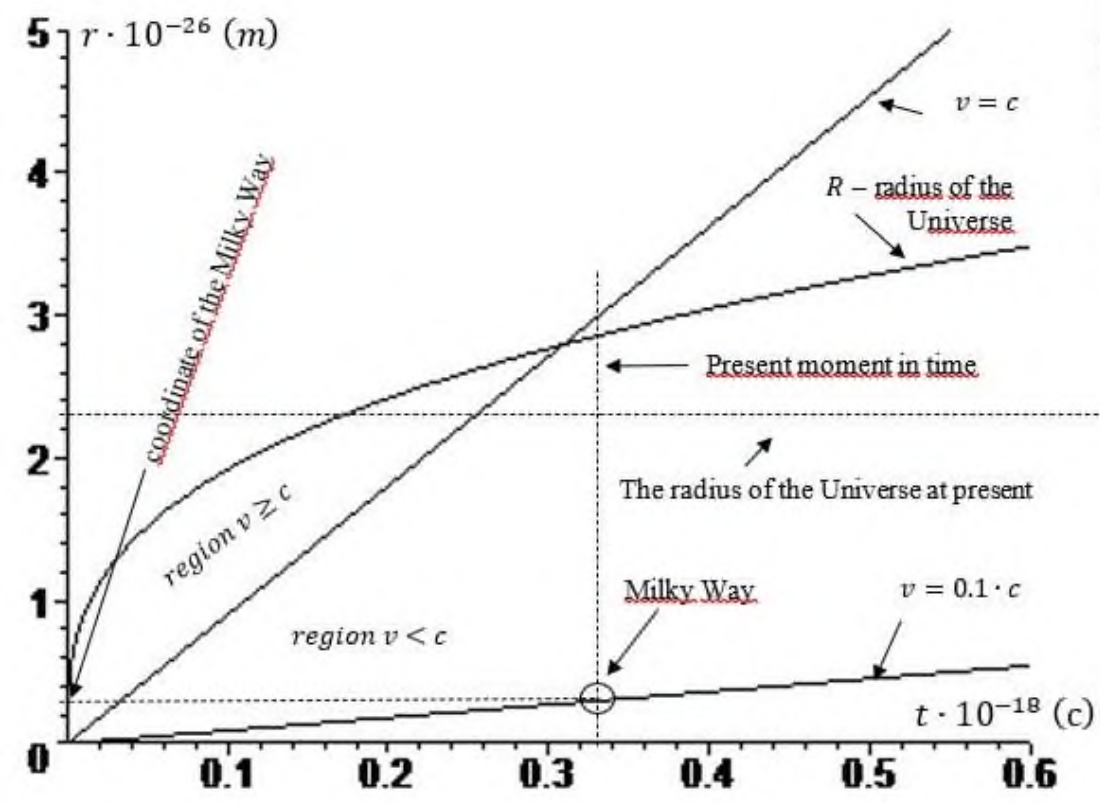

Fig. 4. Division of the Universe into relativistic and non-relativistic regions.

Thus, at present, only the inner regions of the Universe $r<0.1 \cdot R$ are described with sufficient accuracy by the laws of classical physics.

It is now possible to estimate the acceleration of the movement of matter in the Milky Way galaxy at the present time. Indeed, according to what was said above, now the density of matter in the Universe is $\rho=\rho_{0} q=\rho_{0}=10^{-26}(\mathrm{~kg} / \mathrm{m})$, the radius of the Universe at which the galaxy is located is $r=0.3 \cdot 10^{26}(\mathrm{~m})$. Hence, according to $(15), w=-0.3$. $10^{-13}\left(\mathrm{~m} / \mathrm{s}^{2}\right)$. Thus, if ever it is possible to measure acceleration with such precision, then it will be possible to determine the direction to the center of the universe. By the way, the first law of dynamics must be valid with the same precision.

Let us now turn to the energy side of the proposed model of the Universe.

The potential energy of the Universe as a solitary body, i.e. the gravitational potential energy associated with the gravitational attraction of the particles of matter constituting the Universe is determined by the formula:

$$
E_{P}=-\frac{3}{5} G \frac{M^{2}}{R}=-\frac{16 \pi^{2}}{15} G \rho^{2} R^{5}
$$

Substituting the expressions for the incoming quantities, we get

$$
E_{P}=-17,0 \cdot 10^{70} \cdot\left(\frac{1}{-A \tau^{2}+2 C \tau}\right)^{1 / 3}(J)
$$

Comparing it (currently) with the thermal energy of the Universe $E_{T}$ 申e $T=3 \mathrm{~K}$, and assuming that the mass of a particle of matter in the Universe is equal to the mass of a proton $m_{P}=1,67 \cdot 10^{-27}(\mathrm{~kg})$ :

$$
E_{T}=N \frac{3}{2} k T=\frac{M}{m_{0}} \frac{3}{2} T \cdot 1,38 \cdot 10^{-23}=3,7 \cdot 10^{58}(\mathrm{~J})
$$


we make the conclusion that the contribution of thermal energy to the overall energy balance is currently negligible; therefore, it is quite justified to neglect the thermodynamic component in the initial equations.

To calculate the kinetic energy of matter in the Universe $E_{K}$, firstly write down its differential

$$
d E_{K}
$$

in spherical coordinate system:

Integrating, we get

$$
d E_{K}=\rho \frac{v^{2}}{2} 4 \pi r^{2} d r=2 \pi \rho H^{2} r^{4} d r
$$

$$
E_{K}=\frac{2 \pi}{5} \rho H^{2} R^{5}
$$

Substituting the numerical values of the parameters, we find:

$$
E_{K}=0,68 \cdot \frac{(C-A \tau)^{2}}{\left(-A \tau^{2}+2 C \tau\right)^{\frac{4}{3}}} \cdot 10^{70}(\mathrm{~J})
$$

Figure 5 shows graphically the dependence of the kinetic and potential energies of the Universe (pre-multiplied by $10^{-70}$ ) from time to time (solid lines). For clarity, the kinetic energy values are increased by a factor of one hundred. The same graph also shows the third curve, which depicts the time dependence of the sum of kinetic and potential energies (line of dots).

We see that both kinetic and potential energies are minimal during the period of maximum expansion of the Universe. But the potential energy does not reach zero, so during the period of maximum expansion, the density of the substance of the Universe is not zero. The sum of the kinetic and potential energies is zero at $\tau_{1}=0.0598, \tau_{2}=$ 74.940

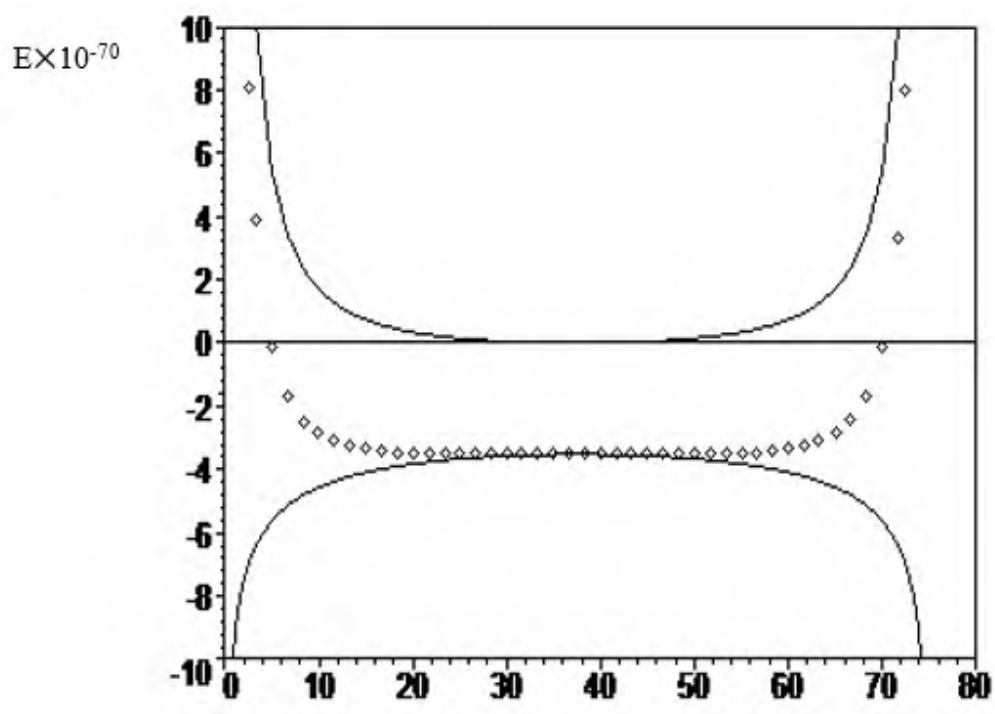

Fig. 5. Time dependence of kinetic EK, potentiallyй EP and total EK+EP energies per cycle of development of the Universe.

But the most interesting thing about these graphs is that the sum of kinetic and potential energies is not constant and not equal to zero. This clearly contradicts the formulation of the energy conservation law in classical physics. 
To understand what is the matter here, it is necessary to remember that in classical physics, potential energy in general and gravitational potential energy in particular are determined with an accuracy up to an additive constant. Moreover, this constant is very freely disposed of, setting it equal to zero and placing this zero at an arbitrary point in space. This is the first thing.

In addition, the existence of "true" gravitational fields is asserted [15]. Further, in classical physics, when determining the gravitational energy of a body, it is stated that "the gravitational energy of a body is not the total energy of the gravitational field, but that part of it that is associated with the gravitational interaction of the particles of the body. It shows how much energy of the gravitational field when the body exists, less than the energy of the gravitational field in its absence "[16].

This definition of the gravitational energy of a body can be interpreted in different ways.

We will understand it in the sense that there is the gravitational energy of the body and the energy of the gravitational field of the Universe, which is the very additive constant. That is, in other words, in the Universe there is a gravitational field that has energy and there is a gravitational field of matter, which reduces the gravitational energy of the field.

The fact is that the dimensions of the solar system, or the Milky Way galaxy, in which we usually apply the law of conservation of energy, are extremely small compared to the scale of the Universe, and within these systems the energy of the gravitational field can indeed be considered constant. The same applies to time scales. High-precision measurements have appeared in the last hundred years, which is absolutely negligible compared to the time scales of the Universe (thousands of billions of years). Therefore, from this point of view, the additive constant is really constant.

But when we consider the Universe as a whole, with its spatial and temporal scales, this additive constant, i.e. the energy of the gravitational field of the universe is changing. It can no longer be considered constant. This energy takes an active part in the exchange of energies.

Most likely, it is this energy of the gravitational field of the Universe that is currently called "dark matter", and the existence of this "dark matter", as we see, was predicted by classical physics (though not entirely explicitly). But we will not use this expression as it is very ambiguous. However, to avoid confusion in the verbal formulations, this field will be called the primary field, in the sense that it already existed at the time of the Big Bang.

In classical physics, when considering the question of the gravitational energy of a material body, nothing is said about kinetic energy. But if the process of spraying a body to "infinity" takes place in a limited time interval, then the speed of the sprayed particles of the body must be finite. That is, these sprayed particles of the body will have kinetic energy that is not taken into account. Note that this kinetic energy is generated not due to the energy of the primary field, but in spite of it, due to the energy of the one who sprays the material body. But although the source of kinetic energy is different, it is generated exactly as much as the energy of the primary field decreases. In this sense, they are interconnected.

In addition, we note that when the body is sprayed to "infinity" the energy of the primary field in the area of the material body will increase, but at "infinity", when the sprayed body is introduced there, the field energy will decrease so that the total energy remains the same. There will only be a redistribution of the energy of the primary field.

As applied to the Universe, it should be noted that, firstly, it will not work to spray to "infinity", since the Universe is a "black hole" and it is impossible to take matter out of its limits. Second, the kinetic energy of the expanding Universe is generated by the energy of the Big Bang. And when formulating the law of conservation of energy for the entire Universe as a whole, it is necessary to take into account this energy of the Big Bang. And 
the question of whether the Universe will expand to "infinity" or not comes down to the question of whether the Big Bang has enough energy to spray the Universe.

The energy of the Big Bang is determined by the kinetic energy of the Universe - EK. The change in the energy of the primary field is determined by the potential energy of the Universe - EP.

In this regard, it becomes necessary to reformulate the law of conservation and transformation of energy in such a way that it can be applied to the entire Universe.

The simplest formulation suggests itself:

The sum of the kinetic energy of the body, its gravitational energy and the energy of the primary field is constant.

Let's designate the energy of the primary field by the symbol EG. Then the law of conservation of energy will be written in the form:

$$
E_{K}+E_{P}+E_{G}=\text { const }
$$

It follows from (21) that energy can pass from one type to another, but so that equality (21) is fulfilled. The energy conservation law in the form (21) automatically turns into the energy conservation law of classical physics, when $E_{G}=$ const, i.e at small sizes, in comparison with the Universe, the area of study and at small time intervals.

Currently, there are still no methods for direct measurement of the primary field energy, but it can be indirectly determined based on equation (21). The additive constant in the conservation law (21) can be determined using various conditions. For example, you can require that at the moment of the greatest expansion of the Universe, the energy of the gravitational field is equal to zero, etc.

From this point of view, a primary field acts on a particle of matter in the Universe, and the gravitational field of the Universe only reduces this primary field.

We apply the operation grad to equation (21) (we omit the sign of the vector, since the direction of the gradient is known):

$$
\operatorname{grad}_{K}+\operatorname{grad} E_{P}+\operatorname{grad} E_{G}=0
$$

We rewrite this equation in the form:

$$
-2 \pi \rho H^{2} r^{4}+\frac{16 \pi^{2}}{3} G \rho^{2} r^{4}=F_{G}
$$

Here $F_{G}$ - the force acting on a particle of matter from the side of the primary field, since the force is equal to the field gradient with the opposite sign. For the Universe not to expand to infinity, this force must be negative, i.e. was directed towards the center of the universe. Therefore, we can write:

$$
2 \pi \rho H^{2} r^{4}-\frac{16 \pi^{2}}{3} G \rho^{2} r^{4} \geq 0
$$

We rewrite this inequality as

$$
\frac{16 \pi^{2}}{3} G \rho r^{4} \cdot\left(\frac{3 H^{2}}{8 \pi G}-\rho\right) \geq 0
$$

Since the factor in front of the bracket is positive, we obtained the condition of the nonsputtering Universe of the Friedmann model $[3,11]$. Here as usual, $\frac{3 H^{2}}{8 \pi G}=\rho_{\text {кр }}-$ critical density of matter in the Universe. Substituting expressions for $\mathrm{H}$ and $\rho$, we obtain condition (22) in the form: 


$$
\begin{gathered}
\frac{3 H^{2}}{8 \pi G \rho} \geq 1 \\
0.04 \cdot \frac{(C-A \tau)^{2}}{-A \tau^{2}+2 C \tau} \geq 1
\end{gathered}
$$

Value $\tau$, satisfying this equality was obtained above: $-\tau_{1}=0.0598, \tau_{2}=74.940$.

Thus, in the initial period of expansion from $\tau=0$, to $\tau=0.0598$ (1,89 billion years) kinetic energy exceeded the energy of the primary field, and the Universe was expanding rapidly. After this moment, the energy of the primary field begins to exceed the kinetic energy and the expansion of the Universe is slowed down, but by inertia it continues to expand until the middle of the life cycle of the Universe. Then it will begin to shrink again.

\section{Conclusion}

Thus, we have built a nonrelativistic model of a cyclic Universe, which allows us to determine the dependence of its global parameters on time, thereby predicting the development of the Universe in the future, expanding our understanding of the Universe in which we live.

The proposed model does not contradict the modern $\Lambda$ CDM model due to the fact that the spatio-temporal areas of applicability of the models are different. If the $\Lambda \mathrm{CDM}$ the model describes the Universe at the earliest stage of expansion (and, accordingly, should describe the Universe at the latest stage of contraction), then the proposed model describes the Universe from about the middle of the expansion period $\tau=1$ to the middle of the compression period $\tau=72$, in this case, the entire period of the life of the Universe is $\Delta \tau=73 \approx 2315$ billion years. But the proposed model is much more "transparent" and clearer for perception.

\section{References}

1. Alexander D. (ed.) The Isaac Newton Guidebook Faraday Institute Publishing (2012)

2. Einstein: The Man, the Genius, and the Theory of Relativity Hardcover - Illustrated (2018)

3. O'Raifeartaigh, C.; McCann, B., "Einstein's cosmic model of 1931 revisited: An analysis and translation of a forgotten model of the universe". The European Physical Journal H. 39, 63-85 (2014)

4. Carroll, Sean; Kaku, Michio, "End of the Universe". How the Universe Works. Discovery Channel (2014)

5. Carroll, B.W.; Ostlie, D. A., An Introduction to Modern Astrophysics (International ed.). Pearson (2013) ISBN 978-1-292-02293-2.

6. Weinberg, S., Dreams of a Final Theory: The Scientist's Search for the Ultimate Laws of Nature. Knopf Doubleday Publishing Group. ISBN 978-0-307-78786-6.

7. Ellis G. F, "Does the Multiverse Really Exist?". Scientific American 305 (2): 38-43 (2011) Bibcode:2011SciAm.305a..38E. doi:10.1038/scientificamerican0811-38. PMID 21827123.

8. E. Chaisson, "First few minutes" Havard Smithsonian Center for Astrophysics (2016)

9. F. Zwicky. «On the Masses of Nebulae and of Clusters of Nebulae» (1937) 
10. Marov, M.Ya., "The Structure of the Universe". The Fundamentals of Modern Astrophysics 279-294 (2015) doi:10.1007/978-1-4614-8730-2_10. ISBN 978-1-46148729-6

11. C. Karahan, A. Altas, D. Demir, Scalars, vectors and tensors from metric-affine gravity, General Relativity and Gravitation 45, 319-343 (2013)

12. M. Lilley, P. B. Lilje, M. Liguori, A. Lewis, F. Levrier. Planck 2018 results. VI. Cosmological parameters (2018)

13. Marov, M. Ya., "The Structure of the Universe". The Fundamentals of Modern Astrophysics 279-294 (2015) doi:10.1007/978-1-4614-8730-2_10. ISBN 978-1-46148729-6

14. G. Sardanashvily, Classical gauge gravitation theory, Int. J. Geom. Methods Mod. Phys. 8,1869-1895 (2011)

15. V. Vitagliano, T. Sotiriou, S. Liberati, The dynamics of metric-affine gravity, Annals of Physics 326, 1259-1273 (2011) 